Sharif University of Technology
Scientia Iranica
Transactions E: Industrial Engineering
hCIENTIA

\title{
On designing CUSUM charts using ratio-type estimators for monitoring the location of normal processes
}

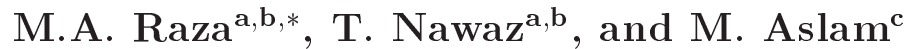 \\ a. School of Mathematical Sciences, Shanghai Jiao Tong University, Shanghai 200240, China. \\ b. Department of Statistics, Government College University Faisalabad, Faisalabad 38000, Pakistan. \\ c. Department of Statistics, Faculty of Sciences, King Abdulaziz University, Jeddah 21551, Saudi Arabia. \\ Received 10 February 2018; received in revised form 13 September 2018; accepted 27 October 2018
}

\section{KEYWORDS}

Auxiliary variable; Control charts; CUSUM statistic; Fast Initial Response (FIR);

Ratio estimator; Average run length; Extra quadratic loss.

\begin{abstract}
A control chart is an important tool in statistical process control that plays a significant role in monitoring and identifying variations in production processes. The Shewhart, the cumulative sum (CUSUM), and the Exponentially Weighted Moving Average (EWMA) control charts are commonly used for detecting process shifts. The CUSUM and the EWMA control charts are more sensitive in detecting smaller shifts, whereas the typical Shewhart chart is sensitive to large process shifts. The present study incorporates ratiotype estimators of the population mean based on auxiliary information in the CUSUM charting structure for monitoring the location of the normal processes. These estimators are more efficient than simple mean estimator in the presence of a high correlation between the study and the auxiliary variables. The Average Run Length (ARL), the standard deviation of run length, and the extra quadratic loss are used to measure the performance of the proposed charts. The proposed charts are compared with the existing CUSUM, CUSUM-FIR, and some other auxiliary information-based control charts on the basis of out-of-control ARLs. The comparison reveals the superiority of the suggested charts over the other existing charts. An illustrative example is also provided for the performance evaluation of the proposed charts.

(C) 2020 Sharif University of Technology. All rights reserved.
\end{abstract}

\section{Introduction}

Statistical process control is a combination of different methods that are employed to monitor a process and enhance the quality of the products by reducing variations in the products [1]. Among these methods, the control chart is the most important and frequently

\footnotetext{
*. Corresponding author. Tel.: +8615301610650 E-mail addresses: ali.raza@sjtu.edu.cn (M.A.Raza); tahir.nawaz@sjtu.edu.cn (T.Nawaz); aslam_ravian@hotmail.com (M.Aslam)
}

used method for monitoring the quality of the products, initially developed by Walter A. Shewhart in the early 1920s. A control chart is a graphical display of the quality characteristics, which is used to monitor the process stability and is constructed in such a way that the associated probability of false alarm is very low. The Shewhart control chart, the cumulative sum (CUSUM) control chart by Page [2], and the Exponentially Weighted Moving Average (EWMA) control chart by Roberts [3] are commonly used for monitoring the process variation. The process variation can be classified into common and assignable cause variations. The process is considered to be in-control in the presence of common cause variations, which are 
considered to be the inherent parts of any production process. The classical Shewhart chart is sensitive to large process shifts, as it utilizes only the current sample information and ignores the previous samples information. On the other hand, the CUSUM and the EWMA charts make use of the past and the current sample information, enabling them to be more sensitive to small process shifts.

In the sampling theory, the use of auxiliary information results in a significant reduction in the variance of the estimators of the population parameters. The auxiliary variable is not the variable of interest, but is instead used to improve the sampling plan or enhance the precision of the estimators. The ratio, product, and regression type estimators are extensively used for estimating the unknown population parameter, provided that a significant correlation between the study and auxiliary variables exists [4]. Different ratio-type estimators are available in the literature for improving the precision of location estimators. For more details, the readers can refer to [5-12] and the references cited therein.

The auxiliary information is also used in the control charting structure in order to improve the sensitivity of the control chart. Zhang [13] proposed the cause-selecting control chart by regressing the study variable on the auxiliary variable. Later, Riaz [14] proposed the Shewhart-type control chart for monitoring process location based on the regression estimator of mean, which incorporates the auxiliary information and has an attractive detection ability, as compared to existing charts. In the same context, Riaz [15] introduced the idea of using the regressiontype estimator of variance based on an auxiliary variable in the control charting structure for improved monitoring of process dispersion. Few researchers have presented their ideas in the context of utilizing auxiliary information in control charting structures (see [16-19]). The product-difference type estimator of the mean was used by Lee et al. [20] for process monitoring under repetitive sampling. It is based on inner and outer control limits so that repetitive sampling is allowed when the monitoring statistic lies between the inner and outer limits. The resulting chart is the extension and improved form of the Mcontrol chart proposed by Riaz [21] by devising the two pair of limits. It is worth mentioning that most of the existing charts based on auxiliary information are Shewhart-type control charts such as [14,17,2022]. Some authors have recently suggested EWMAtype control charts based on auxiliary information (see [23-27]). Recently, Sanusi et al. [28] proposed combined Shewhart CUSUM charts (CSC) based on auxiliary information by incorporating various types of ratio and regression estimators to monitor the process location. The strategy used by them is complicated for the practitioners as they combined the Shewhart and the CUSUM charts besides using various complex estimators of the population mean (specifically the last five estimators considered in their study).

In this article, five relatively simple and efficient auxiliary information based ratio-type estimators of the study variable are used to estimate the process location. The traditional ratio estimator proposed by Cochran [29], the estimators based on quartiles suggested by Al-Omari [8], and a new class of estimators proposed by Singh et al. [10] are used here. Since all these estimators are more efficient than the simple mean estimator in the presence of a moderate to high correlation between the study and the auxiliary variables $[8,10]$, their incorporation in different charting schemes would result in more efficient control charts. The present study integrates the above-mentioned estimators in the CUSUM charting structure with the motivation to further enhance the sensitivity of the chart without complicating the charting structure. The performance of the proposed charts is evaluated in terms of Average Run Length (ARL) and the Standard Deviation of Run Length ( SDRL). The Extra Quadratic Loss (EQL) is also used to measure the overall performance of the proposed charts over a range of shifts.

The rest of this paper is organized as follows: Section 2 presents the structures and properties of the ratio-type location estimators based on the auxiliary information. Section 3 presents the general structures of the proposed CUSUM charts. Performance evaluations of the proposed charts are given in Section 4. Comparison of the proposed with existing control charts and an illustrative example are provided in Section 5. Finally, some conclusions are drawn in Section 6 .

\section{Location estimators based on auxiliary information and their properties}

It is assumed that the target variable $(Y)$ and the auxiliary variable $(X)$ follow the bivariate normal distribution with means $\mu_{Y}$ and $\mu_{X}$, standard deviations $\sigma_{Y}$ and $\sigma_{X}$, and the correlation coefficient $\rho_{X Y}$. Herein, $\left(X_{1}, Y_{1}\right),\left(X_{2}, Y_{2}\right), \cdots,\left(X_{n}, Y_{n}\right)$ is a bivariate random sample with density function $f(x, y)$, the sample statistics based on the measurements of $Y$ and $X$ are defined as $\bar{y}$ and $\bar{x}$ for the means, respectively, $s_{y}^{2}$ and $s_{x}^{2}$ are for the variances, and $r_{x y}$ is for the correlation coefficient. Based on the above preliminaries, some efficient estimators $E_{j}(j=1,2, \cdots, 5)$ of the target variable $(Y)$ based on auxiliary information $(X)$ are presented with their respective bias, $B($.$) , and Mean$ Square Error, MSE(.). These estimators differ from each other in terms of their structures and efficiencies.

(i) The ratio estimator. The ratio estimator makes use of the known population information 
of auxiliary variable to improve the weighting from sample values to population estimates and is useful when the correlation between the target and auxiliary variable is high [29]. The ratio estimator is defined as follows:

$$
E_{1}=\bar{y} \frac{\mu_{x}}{\bar{x}}
$$

The bias and MSE of the ratio estimator up to the first degree of approximation are as follows:

$$
\begin{aligned}
& B\left(E_{1}\right)=\lambda \mu_{y}\left(C_{X}^{2}-\rho_{x y} C_{y} C_{x}\right) \\
& M S E\left(E_{1}\right)=\lambda \mu_{y}^{2}\left(C_{y}^{2}+C_{X}^{2}-2 \rho_{x y} C_{y} C_{x}\right)
\end{aligned}
$$

where $C_{y}=\frac{\sigma_{y}}{\mu_{y}}, C_{x}=\frac{\sigma_{x}}{\mu_{x}}$, and $\lambda=\left(\frac{1-n / N}{n}\right)$.

(ii) The ratio-type estimators suggested by AlOmari [8]. These new types of ratio estimators of the population mean $\mu_{Y}$ are based on either the first quartile $\left(q_{1}\right)$ or the third quartile $\left(q_{3}\right)$ of the auxiliary variable and are defined as follows:

$$
\begin{aligned}
& E_{2}=\bar{y}\left(\frac{\mu_{x}+q_{1}}{\bar{x}+q_{1}}\right), \\
& E_{3}=\bar{y}\left(\frac{\mu_{x}+q_{3}}{\bar{x}+q_{3}}\right) .
\end{aligned}
$$

The bias and MSE up to the first degree of approximation are as follows:

$$
\begin{aligned}
& B\left(E_{2}\right) \cong 0, \quad B\left(E_{3}\right) \cong 0 \\
& M S E\left(E_{2}\right)=\frac{\sigma_{y}^{2}}{n}+\frac{\sigma_{x}^{2}}{n}\left(L_{1}^{2}-2 b_{y x} L_{1}\right) \\
& M S E\left(E_{3}\right)=\frac{\sigma_{y}^{2}}{n}+\frac{\sigma_{x}^{2}}{n}\left(L_{2}^{2}-2 b_{y x} L_{2}\right)
\end{aligned}
$$

where $b_{y x}=\rho_{x y} \frac{\sigma_{y}}{\sigma_{x}}, L_{1}=\frac{\mu_{y}}{\mu_{x}+q_{1}}$, and $L_{2}=\frac{\mu_{y}}{\mu_{x}+q_{3}}$.

(iii) Ratio and product estimator. Singh et al. [10] suggested the following class of estimators of the population mean $\mu_{y}$ using simple random sampling:

$$
\hat{\bar{Y}}=\bar{y}\left[\frac{a \mu_{x}+b \bar{x}}{c \bar{x}+d \mu_{x}}\right],
$$

where $a, b, c$, and $d$ are suitable constants and can be either parametric or any real values. The present study includes the following two estimators based on the above class of estimators:

$$
E_{4}=\bar{y}\left(\frac{l^{2} \mu_{x}-C_{x} \bar{x}}{l^{2} \bar{x}-C_{x} \mu_{x}}\right),
$$

where $a=c=l^{2}, b=d=-C_{x}$, and $l=\rho_{x y} \frac{C_{y}}{C_{x}}$.

$$
E_{5}=\bar{y}\left(\frac{\gamma_{2} \mu_{x}-C_{y} \bar{x}}{\gamma_{2} \bar{x}-C_{y} \mu_{x}}\right),
$$

where $a=c=\gamma_{2}, b=d=-C_{y}$, and $\gamma_{2}$ is the coefficient of kurtosis of the auxiliary variable.

The bias and MSE up to the first degree of approximation are as follows:

$$
\begin{aligned}
& B\left(E_{j}\right)=\lambda \mu_{y} C_{x}^{2}\left\{\psi\left(\theta_{1}-l\right)\right\} \\
& \quad \text { for } \quad j=4,5 \\
& M S E\left(E_{j}\right)=\lambda \mu_{y}^{2}\left\{C_{y}^{2}+\psi C_{x}^{2}(\psi-2 l)\right\} \\
& \quad \text { for } \quad j=4,5
\end{aligned}
$$

where $\psi=\theta_{1}-\theta_{2}, \theta_{1}=c(c+d)^{-1}$, and $\theta_{2}=$ $b(a+b)^{-1}$.

\section{General structure of the proposed CUSUM charts}

The proposed charts are the integration of the estimator $E_{j}$ for $j=1,2, \cdots, 5$ with the CUSUM charting scheme. The CUSUM chart is based on the accumulation of the information of the previous samples in addition to the current sample. For this reason, the CUSUM charts are more effective than the Shewhart charts in detecting small process mean shifts. The present study assumes that the quality characteristic of interest $Y$ and the auxiliary variable $X$ follow the bivariate normal distribution, $(Y, X) \sim$ $N\left(\mu_{y}, \mu_{x}, \sigma_{y}^{2}, \sigma_{x}^{2}, \rho_{x y}\right)$, to define new efficient CUSUM control charts based on location estimators $E_{j}$ for $j=1,2, \cdots, 5$.

Let $Z_{j t}=\frac{E_{j t}-\mu_{E_{j}}}{\sigma_{E_{j}}}$ be the standardized form of the $j$ th estimator in the $t$ th subgroup $(j=1,2, \cdots, 5)$, where $\mu_{E_{j}}=\mu_{y}+B\left(E_{j}\right)$ and $\sigma_{E_{j}}^{2}=\operatorname{MSE}\left(E_{j}\right)$. The CUSUM monitoring statistics under the ratio type location estimators $E_{j}$ based on auxiliary information are defined as follows:

$$
\left\{\begin{array}{l}
M_{j t}^{+}=\max \left[0, Z_{j t}-k+M_{j(t-1)}^{+}\right] \\
M_{j t}^{-}=\max \left[0,-Z_{j t}-k+M_{j(t-1)}^{-}\right]
\end{array}\right.
$$

The statistics $M_{j t}^{+}$and $M_{j t}^{-}$are called the upper and lower CUSUM under the $j$ th estimator, respectively, and the initial values of these monitoring statistics are set equal to zero, i.e., $M_{0}^{+}=M_{0}^{-}=0$. The chart is designed by choosing the two-parameter values, i.e., the reference value, $k$, and the decision interval, $H$. These values are chosen such that a specific in-control ARL is attained. A process is considered to be out of control if either $M_{j t}^{+}$or $M_{j t}^{-}$exceeds the decision interval, $H_{j}$. 
The mean and variance of the ratio-type estimators $E_{j}$, the value of the decision interval $H_{j}$ at a fixed in-control $A R L_{0}$ under the different values of $k$ and sample size $n$, and, subsequently, the out-of-control Average Run Lengths $\left(A R L_{1}\right)$ are obtained through simulation by using the following algorithm.

\subsection{Algorithm}

1. Sample means and variances of the proposed estimators $E_{j}(j=1,2, \cdots, 5)$.

(1.1) Generate 100,000 samples (subgroups) of size $n$, each from the in-control bivariate normal process having the specified parameters;

(1.2) Calculate $E_{j}(j=1,2, \cdots, 5)$ for each subgroup;

(1.3) Calculate mean $\left(E_{j}\right)$, bias $\left(E_{j}\right)$, and MSE $\left(E_{j}\right)$ from 100,000 subgroups.

2. Set up control limits.

(2.1) Generate a random sample of size $n$ from the in-control bivariate normal process, i.e., $(Y, X) \sim N\left(\mu_{y}, \mu_{x}, \sigma_{y}^{2}, \sigma_{x}^{2}, \rho_{x y}\right) ;$

(2.2) Calculate $E_{j}(j=1,2, \cdots, 5)$;

(2.3) Calculate $Z_{j t}$ based on the information generated in step 1.3;

(2.4) Calculate the CUSUM monitoring statistics $M_{j t}^{+}$and $M_{j t}^{-}$;

(2.5) Choose the reference value, $k$, and decision interval, $H$, for desired $A R L_{0}$;

(2.6) Repeat steps 2.1-2.4 $m$ times (say 100,000 times) to compute the in-control ARL. If the in-control ARL is equal to the desired $A R L_{0}$, then move to step 3 with the chosen value of $H$. Otherwise, revise the value of $H$ in order to achieve the desired $A R L_{0}$.

3. Evaluate out-of-control ARLs.

(3.1) Generate a random sample of size $n$ from the shifted bivariate normal process, i.e., $(Y, X) \sim N\left(\mu_{y 0}+\delta \sigma_{y}, \mu_{x}, \sigma_{y}^{2}, \sigma_{x}^{2}, \rho_{x y}\right)$, where $\delta$ is a magnitude of the shift in terms of standard deviation;
(3.2) Repeat Steps 2.2-2.4 $m$ times and calculate the out-of-control ARL and SDRL under different mean shifts using the same value of $H$ obtained in step 2.6;

(3.3) Calculate the EQL in order to evaluate the overall performance of the chart.

\section{Performance evaluation of the proposed charts}

This section presents the performance evaluation of the proposed CUSUM charts by using the (ARL) and the SDRL for different shifts $(\delta)$ in the process through Monte Carlo simulations since it is more accurate and flexible to handle various scenarios than approximation methods [30]. Moreover, to gauge the overall efficiency of the charts based on all the shifts, $E Q L$ is used as a performance indicator. All the simulations are carried out in R software [31]. The ARL is a popular measure for gauging the effectiveness of the control chart in detecting the shift in process. It is defined as the average number of sample points plotted until a plotted sample point indicates an out-of-control signal [1]. Under a fixed $A R L_{0}$, the chart having the smaller $A R L_{1} \mathrm{~s}$ is considered to be superior in detecting process shifts. Similarly, the smaller SDRL value also indicates the superiority of the control chart to detect a shift in the process being monitored [32]. The EQL is defined as the weighted average ARL based on all the shifts used in a control process. The algebraic expression for EQL is as follows:

$$
E Q L=\frac{1}{\delta_{\max }-\delta_{\min }} \int_{\delta_{\min }}^{\delta_{\max }} \delta^{2} A R L(\delta) d \delta,
$$

where $\delta_{\max }$ and $\delta_{\min }$ are the maximum and minimum values of the shifts considered in a process, and $A R L(\delta)$ denotes the ARL at a specific shift. More details can be found in [32-35]. Numerical integration is used to compute the values of EQL.

Table 1 shows the estimated MSE of the estimators $E_{j}(j=1,2, \cdots, 5)$ for various levels of correlation

Table 1. The estimated Mean Square Errors (MSEs) of the estimators $E_{j}(j=1,2, \cdots, 5)$.

\begin{tabular}{ccccccc}
\hline \multirow{2}{*}{$\boldsymbol{n}$} & \multirow{2}{*}{$\boldsymbol{\rho}_{\boldsymbol{x} \boldsymbol{y}}$} & \multicolumn{5}{c}{ Mean square error } \\
\cline { 3 - 7 } & & $\boldsymbol{E}_{\mathbf{1}}$ & $\boldsymbol{E}_{\mathbf{2}}$ & $\boldsymbol{E}_{\mathbf{3}}$ & $\boldsymbol{E}_{\mathbf{4}}$ & $\boldsymbol{E}_{\mathbf{5}}$ \\
\hline \multirow{3}{*}{$\mathbf{5}$} & $\mathbf{0 . 5 0}$ & 0.199908 & 0.150817 & 0.150819 & 0.217996 & 0.201333 \\
& $\mathbf{0 . 6 0}$ & 0.159466 & 0.129884 & 0.130021 & 0.169734 & 0.161048 \\
& $\mathbf{0 . 7 5}$ & 0.100143 & 0.100079 & 0.100418 & 0.103869 & 0.100661 \\
& $\mathbf{0 . 9 0}$ & 0.039643 & 0.069729 & 0.070269 & 0.040981 & 0.040133 \\
& & & & & & \\
\multirow{2}{*}{$\mathbf{1 0}$} & $\mathbf{0 . 5 0}$ & 0.100273 & 0.075222 & 0.075222 & 0.107831 & 0.099609 \\
& $\mathbf{0 . 6 0}$ & 0.080092 & 0.064615 & 0.064684 & 0.085619 & 0.081225 \\
& $\mathbf{0 . 7 5}$ & 0.04994 & 0.049773 & 0.049941 & 0.052022 & 0.050412 \\
& $\mathbf{0 . 9 0}$ & 0.019966 & 0.035002 & 0.035273 & 0.020473 & 0.020046 \\
\hline
\end{tabular}


Table 2. The run length characteristics of the proposed charts when $\rho_{x y}=0.50, k=0.50$, and $n=5$.

\begin{tabular}{|c|c|c|c|c|c|c|c|c|c|c|}
\hline \multirow{3}{*}{$\begin{array}{l}\text { Shift } \\
\qquad(\delta)\end{array}$} & \multicolumn{2}{|c|}{$E_{1}$} & \multicolumn{2}{|c|}{$E_{2}$} & \multicolumn{2}{|c|}{$E_{3}$} & \multicolumn{2}{|c|}{$E_{4}$} & \multicolumn{2}{|c|}{$E_{5}$} \\
\hline & \multicolumn{2}{|c|}{$H=4.780$} & \multicolumn{2}{|c|}{$H=4.754$} & \multicolumn{2}{|c|}{$H=4.756$} & \multicolumn{2}{|c|}{$H=4.785$} & \multicolumn{2}{|c|}{$H=4.772$} \\
\hline & ARL & SDRL & ARL & SDRL & ARL & SDRL & ARL & SDRL & ARL & SDRL \\
\hline 0.00 & 369.89 & 362.53 & 370.55 & 368.21 & 371.47 & 363.49 & 369.88 & 367.68 & 369.20 & 363.82 \\
\hline 0.05 & 264.69 & 259.59 & 244.24 & 239.7 & 247.32 & 237.18 & 276.96 & 273.58 & 268.46 & 261.98 \\
\hline 0.10 & 139.25 & 131.75 & 115.68 & 109.38 & 114.43 & 108.02 & 150.37 & 141.38 & 141.18 & 137.31 \\
\hline 0.15 & 74.41 & 67.73 & 57.28 & 51.52 & 57.85 & 51.74 & 81.52 & 74.92 & 75.52 & 68.04 \\
\hline 0.20 & 43.28 & 36.11 & 32.92 & 26.25 & 33.05 & 26.79 & 47.85 & 41.71 & 43.89 & 37.83 \\
\hline 0.25 & 28.16 & 22.09 & 21.63 & 15.93 & 21.34 & 15.25 & 31.13 & 24.93 & 29.05 & 22.92 \\
\hline 0.50 & 8.28 & 4.08 & 6.79 & 3 & 6.78 & 3.06 & 8.86 & 4.48 & 8.39 & 4.09 \\
\hline 0.75 & 4.79 & 1.77 & 3.99 & 1.33 & 4.00 & 1.33 & 5.05 & 1.92 & 4.81 & 1.75 \\
\hline 1.00 & 3.39 & 1.03 & 2.90 & 0.81 & 2.90 & 0.82 & 3.57 & 1.13 & 3.41 & 1.05 \\
\hline 1.50 & 2.25 & 0.52 & 2.00 & 0.4 & 2.00 & 0.4 & 2.33 & 0.57 & 2.25 & 0.53 \\
\hline 2.00 & 1.80 & 0.43 & 1.54 & 0.5 & 1.53 & 0.49 & 1.85 & 0.42 & 1.79 & 0.43 \\
\hline 2.50 & 1.37 & 0.48 & 1.12 & 0.32 & 1.12 & 0.32 & 1.47 & 0.49 & 1.38 & 0.49 \\
\hline 3.00 & 1.08 & 0.27 & 1 & 0.07 & 1 & 0.07 & 1.12 & 0.33 & 1.08 & 0.27 \\
\hline 4.00 & 1 & 0 & 1 & 0 & 1 & 0 & 1 & 0.02 & 1 & 0.01 \\
\hline 5.00 & 1 & 0 & 1 & 0 & 1 & 0 & 1 & 0 & 1 & 0 \\
\hline EQL & \multicolumn{2}{|c|}{5.278} & \multicolumn{2}{|c|}{4.796} & \multicolumn{2}{|c|}{4.794} & \multicolumn{2}{|c|}{5.460} & \multicolumn{2}{|c|}{5.296} \\
\hline
\end{tabular}

Table 3. The run length characteristics of the proposed charts when $\rho_{x y}=0.60, k=0.5$, and $n=5$.

\begin{tabular}{|c|c|c|c|c|c|c|c|c|c|c|}
\hline \multirow{3}{*}{$\begin{array}{l}\text { Shift } \\
(\delta)\end{array}$} & \multirow{2}{*}{\multicolumn{2}{|c|}{$\begin{array}{c}E_{1} \\
H=4.787\end{array}$}} & \multirow{2}{*}{\multicolumn{2}{|c|}{$\begin{array}{c}E_{2} \\
H=4.773\end{array}$}} & \multirow{2}{*}{\multicolumn{2}{|c|}{$\begin{array}{c}E_{3} \\
H=4.771\end{array}$}} & \multirow{2}{*}{\multicolumn{2}{|c|}{$\begin{array}{c}E_{4} \\
H=4.090\end{array}$}} & \multirow{2}{*}{\multicolumn{2}{|c|}{$\begin{array}{c}E_{5} \\
H=4.082\end{array}$}} \\
\hline & & & & & & & & & & \\
\hline & ARL & SDRL & ARL & SDRL & ARL & SDRL & ARL & SDRL & ARL & SDRL \\
\hline 0.00 & 369.62 & 364.52 & 369.29 & 364.03 & 371.8 & 361.48 & 372.12 & 363.96 & 370.65 & 361.04 \\
\hline 0.05 & 243.91 & 233.33 & 227.6 & 217.96 & 226.06 & 218.34 & 267.62 & 262.57 & 263.32 & 260.35 \\
\hline 0.10 & 118.26 & 111.47 & 102.77 & 95.23 & 104.4 & 97.37 & 141.59 & 136.47 & 135.87 & 129.63 \\
\hline 0.15 & 60.36 & 53.45 & 50.82 & 43.84 & 50.13 & 43.21 & 74.25 & 69.31 & 70.13 & 64.99 \\
\hline 0.20 & 34.15 & 27.06 & 28.49 & 22.32 & 28.66 & 22.32 & 42.75 & 37 & 39.99 & 34.7 \\
\hline 0.25 & 22.5 & 16.37 & 18.53 & 12.73 & 18.65 & 12.73 & 27.38 & 22.67 & 24.99 & 20.05 \\
\hline 0.50 & 7.06 & 3.22 & 6.10 & 2.52 & 6.14 & 2.57 & 7.29 & 3.8 & 6.93 & 3.49 \\
\hline 0.75 & 4.16 & 1.39 & 3.68 & 1.17 & 3.68 & 1.2 & 4.07 & 1.57 & 3.91 & 1.44 \\
\hline 1.00 & 2.99 & 0.86 & 2.69 & 0.72 & 2.69 & 0.73 & 2.86 & 0.9 & 2.78 & 0.86 \\
\hline 1.50 & 2.05 & 0.44 & 1.91 & 0.39 & 1.89 & 0.39 & 1.92 & 0.48 & 1.89 & 0.48 \\
\hline 2.00 & 1.62 & 0.49 & 1.38 & 0.48 & 1.38 & 0.48 & 1.43 & 0.49 & 1.39 & 0.49 \\
\hline 2.50 & 1.17 & 0.37 & 1.04 & 0.21 & 1.04 & 0.21 & 1.08 & 0.27 & 1.06 & 0.23 \\
\hline 3.00 & 1.01 & 0.11 & 1 & 0.03 & 1 & 0.03 & 1 & 0.06 & 1 & 0.04 \\
\hline 4.00 & 1 & 0 & $\mathbf{1}$ & 0 & $\mathbf{1}$ & 0 & 1 & 0 & 1 & 0 \\
\hline 5.00 & 1 & 0 & 1 & 0 & 1 & 0 & 1 & 0 & 1 & 0 \\
\hline EQL & \multicolumn{2}{|c|}{4.89} & \multicolumn{2}{|c|}{4.613} & \multicolumn{2}{|c|}{4.609} & \multicolumn{2}{|c|}{4.849} & \multicolumn{2}{|c|}{4.775} \\
\hline
\end{tabular}

coefficient and different sample sizes. The values of ARL, SDRL, and EQL of the proposed control charts for a sample of size $n=5$ and $k=0.50$ are given in Tables 2-5 at different levels of the correlation coefficient $\left(\rho_{x y}\right)$ for various shifts. On the other hand, the ARL, SDRL, and EQL values for a sample of size $n=10$ and $k=0.50$ at various levels of the correlation coefficient $\left(\rho_{x y}\right)$ are presented in Tables $6-9$ for a range of shifts. To highlight the best chart, the ARL values are written in bold font against each magnitude of shifts.
The main findings regarding the proposed charts based on the results given in Tables 2-9 are summarized as follows:

(i) For a sample of size $n=5$ and given the moderate correlation between the study and the auxiliary variable, i.e., $\rho_{x y}=0.5,0.6$, the suggested CUSUM control charts based on the estimators $E_{2}$ and $E_{3}$ have smaller $A R L_{1} \mathrm{~s}$ than other proposed charts (cf., Tables 2 and 3 ). These 
Table 4. The run length characteristics of the proposed charts when $\rho_{x y}=0.75, k=0.5$, and $n=5$.

\begin{tabular}{|c|c|c|c|c|c|c|c|c|c|c|}
\hline \multirow{3}{*}{$\begin{array}{c}\text { Shift } \\
(\delta)\end{array}$} & \multicolumn{2}{|c|}{$E_{1}$} & \multicolumn{2}{|c|}{$E_{2}$} & \multicolumn{2}{|c|}{$E_{3}$} & \multicolumn{2}{|c|}{$E_{4}$} & \multicolumn{2}{|c|}{$E_{5}$} \\
\hline & \multicolumn{2}{|c|}{$H=4.768$} & \multicolumn{2}{|c|}{$H=4.765$} & \multicolumn{2}{|c|}{$H=4.770$} & \multicolumn{2}{|c|}{$H=3.341$} & \multicolumn{2}{|c|}{$H=3.339$} \\
\hline & ARL & SDRL & ARL & SDRL & ARL & SDRL & ARL & SDRL & ARL & SDRL \\
\hline 0.00 & 371.72 & 366.31 & 370.79 & 365.03 & 369.95 & 363.74 & 372.91 & 369.45 & 369.38 & 367.35 \\
\hline 0.05 & 203.56 & 198.99 & 210.46 & 204.71 & 210.47 & 201.33 & 249.79 & 245.89 & 245.11 & 239.56 \\
\hline 0.10 & 83.11 & 76.12 & 83.52 & 76.80 & 84.90 & 77.49 & 119.25 & 118.01 & 115.10 & 111.47 \\
\hline 0.15 & 38.51 & 32.23 & 39.24 & 32.46 & 39.06 & 32.66 & 57.56 & 53.16 & 55.99 & 51.85 \\
\hline 0.20 & 21.82 & 15.82 & 21.86 & 15.79 & 22.06 & 15.89 & 30.83 & 26.66 & 29.89 & 25.61 \\
\hline 0.25 & 14.65 & 9.35 & 14.68 & 9.29 & 14.77 & 9.33 & 18.80 & 15.1 & 18.26 & 14.53 \\
\hline 0.50 & 5.15 & 1.96 & 5.16 & 1.97 & 5.14 & 1.97 & 4.89 & 2.48 & 4.71 & 2.32 \\
\hline 0.75 & 3.16 & 0.93 & 3.17 & 0.93 & 3.18 & 0.93 & 2.79 & 0.99 & 2.72 & 0.97 \\
\hline 1.00 & 2.35 & 0.59 & 2.36 & 0.57 & 2.36 & 0.58 & 2.02 & 0.61 & 1.98 & 0.59 \\
\hline 1.50 & 1.71 & 0.46 & 1.70 & 0.46 & 1.70 & 0.46 & 1.29 & 0.45 & 1.26 & 0.44 \\
\hline 2.00 & 1.14 & 0.35 & 1.14 & 0.35 & 1.15 & 0.35 & 1.02 & 0.13 & 1.01 & 0.12 \\
\hline 2.50 & 1 & 0.06 & 1 & 0.07 & 1 & 0.07 & 1 & 0 & 1 & 0 \\
\hline 3.00 & 1 & 0 & 1 & 0 & 1 & 0 & 1 & 0 & 1 & 0 \\
\hline 4.00 & 1 & 0 & 1 & 0 & 1 & 0 & 1 & 0 & 1 & 0 \\
\hline 5.00 & 1 & $\mathbf{0}$ & 1 & 0 & 1 & 0 & 1 & 0 & 1 & 0 \\
\hline EQL & \multicolumn{2}{|c|}{4.368} & \multicolumn{2}{|c|}{4.371} & \multicolumn{2}{|c|}{4.374} & \multicolumn{2}{|c|}{4.328} & \multicolumn{2}{|c|}{4.301} \\
\hline
\end{tabular}

Table 5. The run length characteristics of the proposed charts when $\rho_{x y}=0.90, k=0.5$, and $n=5$.

\begin{tabular}{|c|c|c|c|c|c|c|c|c|c|c|}
\hline \multirow{3}{*}{$\begin{array}{l}\text { Shift } \\
(\delta)\end{array}$} & \multicolumn{2}{|c|}{$E_{1}$} & \multicolumn{2}{|c|}{$E_{2}$} & \multicolumn{2}{|c|}{$E_{3}$} & \multicolumn{2}{|c|}{$E_{4}$} & \multicolumn{2}{|c|}{$E_{5}$} \\
\hline & \multicolumn{2}{|c|}{$H=4.820$} & \multicolumn{2}{|c|}{$H=4.782$} & \multicolumn{2}{|c|}{$H=4.783$} & \multicolumn{2}{|c|}{$H=2.809$} & \multicolumn{2}{|c|}{$H=2.811$} \\
\hline & ARL & SDRL & ARL & SDRL & ARL & SDRL & ARL & SDRL & ARL & SDRL \\
\hline 0.00 & 370.34 & 367.67 & 370.72 & 362.64 & 371.60 & 365.05 & 370.88 & 368.96 & 370.07 & 374.3 \\
\hline 0.05 & 118.02 & 108.97 & 176.18 & 168.93 & 177.15 & 169.34 & 182.47 & 179.95 & 179.56 & 177.27 \\
\hline 0.10 & 34.92 & 28.33 & 60.75 & 53.56 & 61.37 & 55.3 & 62.58 & 59.9 & 61.03 & 58.25 \\
\hline 0.15 & 16.06 & 10.57 & 27.31 & 20.84 & 27.79 & 21.32 & 24.67 & 21.54 & 24.53 & 21.53 \\
\hline 0.20 & 9.85 & 5.24 & 15.96 & 10.53 & 16.07 & 10.69 & 12.65 & 9.83 & 12.40 & 9.62 \\
\hline 0.25 & 7.03 & 3.18 & 10.81 & 6.02 & 10.95 & 6.13 & 7.69 & 5.17 & 7.56 & 5.00 \\
\hline 0.50 & 3.01 & 0.85 & 4.14 & 1.39 & 4.11 & 1.37 & 2.46 & 0.96 & 2.42 & 0.93 \\
\hline 0.75 & 2.05 & 0.42 & 2.62 & 0.69 & 2.63 & 0.7 & 1.52 & 0.54 & 1.50 & 0.54 \\
\hline 1.00 & 1.62 & 0.48 & 2.04 & 0.41 & 2.04 & 0.42 & 1.11 & 0.31 & 1.10 & 0.3 \\
\hline 1.50 & 1.01 & 0.11 & 1.34 & 0.47 & 1.35 & 0.47 & 1 & 0.02 & 1 & 0 \\
\hline 2.00 & 1 & 0 & 1.01 & 0.11 & 1.01 & 0.1 & 1 & 0 & 1 & 0 \\
\hline 2.50 & 1 & 0 & 1 & 0 & 1 & 0 & 1 & 0 & 1 & 0 \\
\hline 3.00 & 1 & 0 & 1 & 0 & 1 & 0 & 1 & 0 & 1 & 0 \\
\hline 4.00 & 1 & 0 & 1 & 0 & 1 & 0 & 1 & 0 & 1 & 0 \\
\hline 5.00 & 1 & 0 & 1 & 0 & 1 & 0 & 1 & 0 & 1 & 0 \\
\hline EQL & \multicolumn{2}{|c|}{3.912} & \multicolumn{2}{|c|}{4.133} & \multicolumn{2}{|c|}{4.138} & \multicolumn{2}{|c|}{3.907} & \multicolumn{2}{|c|}{3.902} \\
\hline
\end{tabular}

two charts perform almost identically in detecting the shift in the process mean for all magnitudes of shifts. It is indicated that these charts are faster in detecting the smaller shift in the process mean than the other charts. These findings are further supported by smaller SDRL values for these two charts. The overall performance indicator EQL confirms the competitiveness of the control charts based on the estimators $E_{2}$ and $E_{3}$ and their dominance in detecting the shift in the process mean as compared to other proposed charts. Similar results are found for a sample of size $n=10$ (cf., Tables 6 and 7 );

(ii) In the scenario in which the sample size is $n=5$ and, yet, the correlation between the study and auxiliary variable is high, i.e., $\rho_{x y}=0.75,0.90$, the CUSUM control chart based on the estimator $E_{1}$ has smaller $A R L_{1}$ and SDRL values than other suggested charts for the small magnitude 
Table 6. The run length characteristics of the proposed charts when $\rho_{x y}=0.50, k=0.5$, and $n=10$.

\begin{tabular}{|c|c|c|c|c|c|c|c|c|c|c|}
\hline \multirow{3}{*}{$\begin{array}{l}\text { Shift } \\
(\delta)\end{array}$} & \multicolumn{2}{|c|}{$E_{1}$} & \multicolumn{2}{|c|}{$E_{2}$} & \multicolumn{2}{|c|}{$E_{3}$} & \multicolumn{2}{|c|}{$E_{4}$} & \multicolumn{2}{|c|}{$E_{5}$} \\
\hline & \multicolumn{2}{|c|}{$H=4.769$} & \multicolumn{2}{|c|}{$H=4.774$} & \multicolumn{2}{|c|}{$H=4.773$} & \multicolumn{2}{|c|}{$H=4.825$} & \multicolumn{2}{|c|}{$H=4.820$} \\
\hline & ARL & SDRL & ARL & SDRL & ARL & SDRL & ARL & SDRL & ARL & SDRL \\
\hline 0.00 & 369.93 & 363.75 & 369.65 & 368.19 & 369.48 & 355.67 & 371.26 & 365.23 & 370.07 & 364.32 \\
\hline 0.05 & 209.79 & 202.06 & 180.62 & 176.39 & 182.32 & 173.70 & 213.83 & 209.17 & 205.45 & 197.16 \\
\hline 0.10 & 85.88 & 80.33 & 65.04 & 57.95 & 65.91 & 59.92 & 87.96 & 80.12 & 84.65 & 78.70 \\
\hline 0.15 & 39.63 & 33.29 & 29.61 & 22.99 & 29.24 & 22.64 & 42.43 & 35.99 & 39.88 & 33.70 \\
\hline 0.20 & 22.47 & 16.49 & 17.12 & 11.58 & 16.95 & 11.50 & 23.83 & 17.46 & 22.14 & 16.06 \\
\hline 0.25 & 14.98 & 9.58 & 11.39 & 6.48 & 11.58 & 6.61 & 15.80 & 10.31 & 14.83 & 9.47 \\
\hline 0.50 & 5.18 & 1.98 & 4.31 & 1.48 & 4.29 & 1.49 & 5.45 & 2.16 & 5.18 & 1.99 \\
\hline 0.75 & 3.20 & 0.96 & 2.73 & 0.75 & 2.72 & 0.74 & 3.35 & 1.02 & 3.21 & 0.95 \\
\hline 1.00 & 2.37 & 0.59 & 2.09 & 0.44 & 2.09 & 0.44 & 2.47 & 0.63 & 2.39 & 0.59 \\
\hline 1.50 & 1.71 & 0.47 & 1.43 & 0.49 & 1.43 & 0.49 & 1.78 & 0.44 & 1.72 & 0.46 \\
\hline 2.00 & 1.16 & 0.36 & 1.02 & 0.15 & 1.02 & 0.14 & 1.22 & 0.41 & 1.16 & 0.36 \\
\hline 2.50 & 1.01 & 0.07 & 1 & 0 & 1 & 0.01 & 1.01 & 0.11 & 1.01 & 0.07 \\
\hline 3.00 & 1 & 0 & 1 & 0 & 1 & 0 & 1 & 0.01 & 1 & 0 \\
\hline 4.00 & 1 & 0 & 1 & 0 & 1 & 0 & 1 & 0 & 1 & 0 \\
\hline 5.00 & 1 & 0 & 1 & 0 & 1 & 0 & 1 & 0 & 1 & 0 \\
\hline EQL & \multicolumn{2}{|c|}{4.390} & \multicolumn{2}{|c|}{4.178} & \multicolumn{2}{|c|}{4.178} & \multicolumn{2}{|c|}{4.448} & \multicolumn{2}{|c|}{4.391} \\
\hline
\end{tabular}

Table 7. The run length characteristics of the proposed charts when $\rho_{x y}=0.60, k=0.5$, and $n=10$.

\begin{tabular}{|c|c|c|c|c|c|c|c|c|c|c|}
\hline \multirow{3}{*}{$\begin{array}{l}\text { Shift } \\
(\delta)\end{array}$} & \multicolumn{2}{|c|}{$E_{1}$} & \multicolumn{2}{|c|}{$E_{2}$} & \multicolumn{2}{|c|}{$E_{3}$} & \multicolumn{2}{|c|}{$E_{4}$} & \multicolumn{2}{|c|}{$E_{5}$} \\
\hline & \multicolumn{2}{|c|}{$H=4.773$} & \multicolumn{2}{|c|}{$H=4.796$} & \multicolumn{2}{|c|}{$H=4.794$} & \multicolumn{2}{|c|}{$H=4.057$} & \multicolumn{2}{|c|}{$H=4.055$} \\
\hline & ARL & SDRL & ARL & SDRL & ARL & SDRL & ARL & SDRL & ARL & SDRL \\
\hline 0.00 & 370.12 & 356.22 & 369.93 & 362.70 & 369.78 & 365.29 & 369.46 & 360.01 & 370.94 & 363.21 \\
\hline 0.05 & 185.25 & 176.43 & 167.01 & 158.83 & 164.33 & 157.78 & 207.84 & 205.49 & 200.85 & 196.38 \\
\hline 0.10 & 67.54 & 61.63 & 56.00 & 49.81 & 57.52 & 51.15 & 83.05 & 77.71 & 79.88 & 73.88 \\
\hline 0.15 & 31.22 & 24.79 & 25.43 & 19.23 & 25.39 & 19.52 & 37.40 & 32.26 & 35.64 & 30.44 \\
\hline 0.20 & 18.07 & 12.35 & 14.89 & 9.45 & 14.86 & 9.41 & 20.63 & 15.88 & 19.60 & 14.77 \\
\hline 0.25 & 12.07 & 7.10 & 10.13 & 5.38 & 10.20 & 5.46 & 13.33 & 8.85 & 12.69 & 8.25 \\
\hline 0.50 & 4.46 & 1.59 & 3.92 & 1.29 & 3.97 & 1.31 & 4.40 & 1.75 & 4.28 & 1.69 \\
\hline 0.75 & 2.83 & 0.79 & 2.53 & 0.66 & 2.54 & 0.66 & 2.68 & 0.81 & 2.62 & 0.79 \\
\hline 1.00 & 2.15 & 0.47 & 1.98 & 0.39 & 1.98 & 0.39 & 2.03 & 0.50 & 1.98 & 0.50 \\
\hline 1.50 & 1.49 & 0.50 & 1.27 & 0.45 & 1.27 & 0.45 & 1.32 & 0.47 & 1.27 & 0.44 \\
\hline 2.00 & 1.03 & 0.18 & 1.01 & 0.07 & 1.01 & 0.08 & 1.01 & 0.12 & 1.01 & 0.10 \\
\hline 2.50 & 1 & 0.01 & 1 & 0 & 1 & 0 & 1 & 0.01 & 1 & 0 \\
\hline 3.00 & 1 & 0 & 1 & 0 & 1 & 0 & 1 & 0 & 1 & 0 \\
\hline 4.00 & 1 & 0 & 1 & 0 & 1 & 0 & 1 & 0 & 1 & 0 \\
\hline 5.00 & 1 & 0 & 1 & 0 & 1 & 0 & 1 & 0 & 1 & 0 \\
\hline EQL & \multicolumn{2}{|c|}{4.215} & \multicolumn{2}{|c|}{4.094} & \multicolumn{2}{|c|}{4.095} & \multicolumn{2}{|c|}{4.198} & \multicolumn{2}{|c|}{4.168} \\
\hline
\end{tabular}

of shifts, i.e., $\delta \leq 0.25$ (cf., Tables 4 and 5 ). For the moderate to large magnitude of shifts, i.e., $\delta>0.25$, the CUSUM chart based on the estimator $E_{5}$ has smaller $A R L_{1}$ values than other proposed charts. On the overall performance front, the EQL indicates that $E_{5}$ based CUSUM chart enjoys superior efficiency in detecting the shift in the process mean. A similar pattern is observed for the sample of size $n=10$ (cf., Tables 8 and 9 ); (iii) Generally, all the proposed charts almost perform alike in detecting large shifts in the process irrespective of the sample size and the magnitude of the correlation between the study and auxiliary variable (cf., Tables 2-9). Furthermore, the suggested control charts have unbiased ARL since the $A R L_{0}$ remains to be higher than $A R L_{1}$ for all choices of shifts $(\delta)$. Moreover, as the magnitude of shift increases, the ARL and SDRL values approach 1 and 0 , respectively (cf., Tables $2-9$ ). 
Table 8. The run length characteristics of the proposed charts when $\rho_{x y}=0.75, k=0.5$, and $n=10$.

\begin{tabular}{|c|c|c|c|c|c|c|c|c|c|c|}
\hline \multirow{2}{*}{$\begin{array}{l}\text { Shift } \\
(\delta)\end{array}$} & \multicolumn{2}{|c|}{$E_{1}$} & \multicolumn{2}{|c|}{$E_{2}$} & \multicolumn{2}{|c|}{$E_{3}$} & \multicolumn{2}{|c|}{$E_{4}$} & \multicolumn{2}{|c|}{$E_{5}$} \\
\hline & ARL & SDRL & ARL & SDRL & ARL & SDRL & ARL & SDRL & ARL & SDRL \\
\hline 0.00 & 370.48 & 367.01 & 370.28 & 363.58 & 370.62 & 362.35 & 371.19 & 364.48 & 372.33 & 371.03 \\
\hline 0.05 & 140.08 & 133.49 & 142.43 & 135.14 & 143.58 & 137.20 & 188.75 & 185.41 & 183.77 & 180.29 \\
\hline 0.10 & 43.67 & 37.36 & 43.77 & 37.46 & 43.74 & 36.82 & 65.17 & 61.21 & 62.46 & 58.53 \\
\hline 0.15 & 19.88 & 14.47 & 19.89 & 13.82 & 19.98 & 13.97 & 27.50 & 23.90 & 26.58 & 23.07 \\
\hline 0.20 & 11.82 & 6.91 & 11.84 & 6.79 & 11.92 & 6.90 & 14.26 & 10.75 & 13.84 & 10.52 \\
\hline 0.25 & 8.34 & 4.09 & 8.38 & 4.06 & 8.37 & 4.06 & 9.07 & 5.92 & 8.83 & 5.69 \\
\hline 0.50 & 3.41 & 1.04 & 3.39 & 1.02 & 3.38 & 1.04 & 2.99 & 1.13 & 2.95 & 1.09 \\
\hline 0.75 & 2.24 & 0.52 & 2.23 & 0.51 & 2.24 & 0.52 & 1.90 & 0.58 & 1.87 & 0.57 \\
\hline 1.00 & 1.80 & 0.43 & 1.80 & 0.42 & 1.80 & 0.42 & 1.40 & 0.49 & 1.36 & 0.48 \\
\hline 1.50 & 1.08 & 0.27 & 1.07 & 0.25 & 1.08 & 0.26 & 1.01 & 0.07 & 1 & 0.07 \\
\hline 2.00 & 1 & 0.01 & 1 & 0.01 & 1 & 0.02 & 1 & 0 & 1 & 0 \\
\hline 2.50 & 1 & 0 & 1 & 0 & 1 & 0 & 1 & 0 & 1 & 0 \\
\hline 3.00 & 1 & 0 & 1 & 0 & 1 & 0 & 1 & 0 & 1 & 0 \\
\hline 4.00 & 1 & 0 & 1 & 0 & 1 & 0 & 1 & 0 & 1 & 0 \\
\hline 5.00 & 1 & 0 & 1 & 0 & 1 & 0 & 1 & 0 & 1 & 0 \\
\hline EQL & 3. & & & 78 & & & & & & \\
\hline
\end{tabular}

Table 9. The run length characteristics of the proposed charts when $\rho_{x y}=0.90, k=0.5$, and $n=10$.

\begin{tabular}{|c|c|c|c|c|c|c|c|c|c|c|}
\hline \multirow{2}{*}{$\begin{array}{l}\text { Shift } \\
(\delta)\end{array}$} & \multicolumn{2}{|c|}{$E_{1}$} & \multicolumn{2}{|c|}{$E_{2}$} & \multicolumn{2}{|c|}{$E_{3}$} & \multicolumn{2}{|c|}{$E_{4}$} & \multicolumn{2}{|c|}{$E_{5}$} \\
\hline & ARL & SDRL & ARL & SDRL & ARL & SDRL & ARL & SDRL & ARL & SDRL \\
\hline 0.00 & 368.73 & 356.79 & 371.06 & 364.66 & 369.15 & 362.08 & 370.51 & 363.16 & 371.02 & 368.83 \\
\hline 0.05 & 66.39 & 60.24 & 109.51 & 101.12 & 110.89 & 106.85 & 116.71 & 114.00 & 112.27 & 109.31 \\
\hline 0.10 & 17.98 & 12.42 & 30.60 & 23.91 & 30.84 & 24.52 & 28.32 & 25.08 & 28.28 & 25.14 \\
\hline 0.15 & 9.02 & 4.57 & 14.56 & 9.03 & 14.42 & 9.09 & 11.30 & 8.50 & 10.79 & 8.00 \\
\hline 0.20 & 5.87 & 2.40 & 8.89 & 4.47 & 8.94 & 4.58 & 6.01 & 3.64 & 5.89 & 3.53 \\
\hline 0.25 & 4.46 & 1.57 & 6.48 & 2.82 & 6.51 & 2.81 & 4.12 & 2.09 & 4.24 & 2.05 \\
\hline 0.50 & 2.14 & 0.46 & 2.79 & 0.77 & 2.80 & 0.77 & 1.63 & 0.58 & 1.61 & 0.57 \\
\hline 0.75 & 1.49 & 0.50 & 1.95 & 0.39 & 1.96 & 0.39 & 1.07 & 0.26 & 1.06 & 0.24 \\
\hline 1.00 & 1.03 & 0.18 & 1.46 & 0.49 & 1.47 & 0.50 & 1 & 0.01 & 1 & 0.01 \\
\hline 1.50 & 1 & 0 & 1 & 0.06 & 1 & 0.05 & 1 & 0 & 1 & 0 \\
\hline 2.00 & 1 & 0 & 1 & 0 & 1 & 0 & 1 & 0 & 1 & 0 \\
\hline 2.50 & 1 & 0 & 1 & 0 & 1 & 0 & 1 & 0 & 1 & 0 \\
\hline 3.00 & 1 & 0 & 1 & 0 & 1 & 0 & 1 & 0 & 1 & 0 \\
\hline 4.00 & 1 & 0 & 1 & 0 & 1 & 0 & 1 & 0 & 1 & 0 \\
\hline 5.00 & 1 & 0 & 1 & 0 & 1 & 0 & 1 & 0 & 1 & 0 \\
\hline EQL & \multicolumn{2}{|c|}{3.783} & \multicolumn{2}{|c|}{3.882} & \multicolumn{2}{|c|}{3.884} & \multicolumn{2}{|c|}{3.773} & \multicolumn{2}{|c|}{3.769} \\
\hline
\end{tabular}

\section{Comparison of the proposed and existing control charts}

The performance of the proposed CUSUM charts is compared with that of some existing control charts such as the CUSUM chart introduced by Roberts [3], MeanFIR control chart suggested by Lucas and Crosier [36], robust CUSUM charts based on median, Mid-Range (MR), and Hodges-Lehmann (HL) and Tri-Mean (TM) suggested by Nazir et al. [37]. Moreover, the per- formance of the proposed charts is also evaluated in comparison to the auxiliary information based M-type control chart introduced by Riaz [21], the modification of M-type charts by using repetitive sampling proposed by Lee et al. [20], and the combined Shewhart CUSUM (CSC) charts suggested by Sanusi et al. [28].

When $n=5, k=0.5, \rho_{x y}=0.75$, and $A R L_{0}=$ 370 , the proposed auxiliary information based CUSUM control charts using $E_{1}, E_{2}$, and $E_{3}$ considerably outperform the existing CSC control charts for small to 
Table 10. The Average Run Length (ARLs) comparison of the proposed CUSUM charts and the existing combined Shewhart CUSUM (CSC) chart suggested by Sanusi et al. [28] when $n=5, \rho=0.75$, and $k=0.5$ at $A R L_{0}=370$.

\begin{tabular}{|c|c|c|c|c|c|c|c|c|c|c|c|c|}
\hline \multirow{2}{*}{$\begin{array}{l}\text { Chart } \\
\text { type }\end{array}$} & \multirow{2}{*}{ Estimator } & \multicolumn{11}{|c|}{ Shift $(\delta)$} \\
\hline & & 0.00 & 0.05 & 0.10 & 0.15 & 0.20 & 0.25 & 0.50 & 0.75 & 1.00 & 1.50 & 2.00 \\
\hline \multirow{10}{*}{ 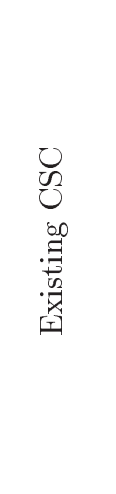 } & $M_{1}$ & 370.04 & 288.21 & 163.42 & 86.64 & 50.12 & 31.69 & 8.85 & 4.89 & 3.26 & 1.65 & 1.11 \\
\hline & $M_{2}$ & 370.12 & 272.48 & 145.40 & 69.90 & 36.74 & 22.81 & 7.06 & 4.17 & 2.94 & 1.59 & 1.07 \\
\hline & $M_{3}$ & 370.53 & 240.41 & 99.71 & 45.52 & 25.19 & 16.23 & 5.36 & 2.94 & 1.85 & 1.06 & 1.00 \\
\hline & $M_{4}$ & 371.48 & 231.62 & 97.28 & 44.83 & 25.05 & 16.43 & 5.34 & 2.90 & 1.80 & 1.06 & 1.00 \\
\hline & $M_{5}$ & 369.85 & 260.53 & 125.32 & 56.28 & 30.37 & 20.58 & 6.86 & 4.07 & 2.84 & 1.42 & 1.03 \\
\hline & $M_{6}$ & 370.03 & 312.25 & 229.14 & 146.34 & 90.65 & 59.18 & 14.40 & 7.55 & 5.08 & 3.09 & 2.05 \\
\hline & $M_{7}$ & 370.83 & 322.73 & 232.18 & 147.78 & 92.51 & 61.03 & 14.40 & 7.62 & 5.14 & 3.10 & 2.07 \\
\hline & $M_{8}$ & 368.23 & 309.06 & 217.54 & 136.90 & 84.03 & 53.63 & 13.30 & 7.13 & 4.84 & 2.98 & 2.03 \\
\hline & $M_{9}$ & 371.10 & 326.80 & 236.50 & 152.58 & 94.49 & 60.57 & 14.53 & 7.63 & 5.17 & 3.12 & 2.09 \\
\hline & $M_{10}$ & 369.85 & 286.99 & 154.56 & 75.70 & 40.75 & 25.08 & 7.57 & 4.43 & 3.16 & 1.76 & 1.12 \\
\hline \multirow{5}{*}{ 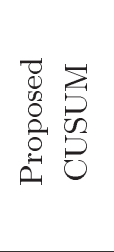 } & $E_{1}$ & 371.72 & 203.56 & 83.11 & 38.51 & 21.82 & 14.65 & 5.15 & 3.16 & 2.35 & 1.71 & 1.14 \\
\hline & $E_{2}$ & 370.79 & 210.46 & 83.52 & 39.24 & 21.86 & 14.68 & 5.16 & 3.17 & 2.36 & 1.7 & 1.14 \\
\hline & $E_{3}$ & 369.95 & 210.47 & 84.9 & 39.06 & 22.06 & 14.77 & 5.14 & 3.18 & 2.36 & 1.7 & 1.15 \\
\hline & $E_{4}$ & 372.91 & 249.79 & 119.25 & 57.56 & 30.83 & 18.8 & 4.89 & 2.79 & 2.02 & 1.29 & 1.02 \\
\hline & $E_{5}$ & 369.38 & 245.11 & 115.1 & 55.99 & 29.89 & 18.26 & 4.71 & 2.72 & 1.98 & 1.26 & 1.01 \\
\hline
\end{tabular}

Table 11. The Average Run Length (ARLs) comparison of the proposed charts $E_{j}(j=1,2, \cdots, 5)$ and Riaz [21] and Lee et al. [20] M-type charts based on auxiliary information when $n=20, \rho_{x y}=0.50$ at $A R L_{0}=220$.

\begin{tabular}{|c|c|c|c|c|c|c|c|}
\hline Shift & $E_{1}$ & $E_{2}$ & $E_{3}$ & $E_{4}$ & $E_{5}$ & M-chart & $\begin{array}{l}\text { M-chart under } \\
\text { rep. sampling }\end{array}$ \\
\hline$(\delta)$ & $H=4.264$ & $H=4.277$ & $H=4.266$ & $H=4.269$ & $H=4.261$ & $k=6.128$ & $\begin{array}{l}k_{1}=5.7 \\
k_{2}=4.3\end{array}$ \\
\hline 0.00 & 221.28 & 220.94 & 219.22 & 222.85 & 221.36 & 223.4 & 223.6 \\
\hline 0.10 & 36.32 & 27.09 & 27.04 & 39.7 & 35.84 & 99.3 & 97.7 \\
\hline 0.20 & 10.91 & 8.43 & 8.32 & 11.28 & 10.67 & 29.9 & 28.8 \\
\hline 0.30 & 5.83 & 4.78 & 4.76 & 6.21 & 5.83 & 10.8 & 10.0 \\
\hline 0.40 & 4.04 & 3.37 & 3.37 & 4.26 & 4.05 & 4.8 & 4.4 \\
\hline 0.50 & 3.1 & 2.65 & 2.64 & 3.27 & 3.1 & 2.6 & 2.4 \\
\hline 1.00 & 1.62 & 1.35 & 1.34 & 1.7 & 1.62 & 1 & 1 \\
\hline 1.50 & 1.03 & 1 & 1 & 1 & 1 & 1 & 1 \\
\hline 2.00 & 1 & 1 & 1 & 1 & 1 & 1 & 1 \\
\hline
\end{tabular}

moderate shifts in the process mean, i.e., $\delta<0.75$ (cf., Table 10). For large shifts $(\delta>0.75)$, the existing CSC and proposed CUSUM charts almost perform alike as the differences in ARLs are negligible. A similar comparison can be made for various combinations of $n, k$, and $\rho_{x y}$. A comparison between the proposed CUSUM charts and M-Type control charts of Riaz [21] and Lee et al. [20] is made for $n=20$ and $\rho_{x y}=0.50$ at $A R L_{0}=220$. Riaz [21] did not report the ARL performance of his chart; however, Lee et al. [20] provided the ARL comparison between their chart and that of Riaz [21]. The suggested charts have shown a better shift detection ability in the process mean than the auxiliary information based M-Type control charts proposed by Riaz [21] and Lee et al. [20]. Particularly for the smaller magnitude of shifts, the performance of the suggested CUSUM charts is very dominating (cf., Table 11).

To further support the above findings, the ARL curves of the three of our suggested CUSUM control charts based on $E_{1}, E_{2}$, and $E_{5}$ are drawn against the existing CUSUM charts. We have not included $E_{3}$ and $E_{4}$ since the performances of $E_{2}$ and $E_{3}$ are almost the same as those of $E_{4}$ and $E_{5}$. Figure 1 depicts 


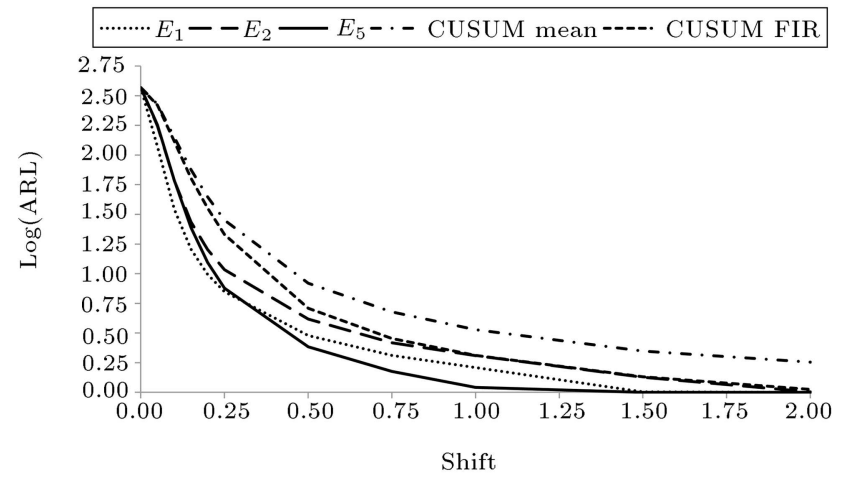

Figure 1. Average Run Length (ARL) curves of the proposed CUSUM charts using $E_{j}(j=1,2,5)$ and the existing CUSUM mean and FIR CUSUM charts when $n=5, k=0.5$, and $\rho_{x y}=0.90$ at $A R L_{0}=370$.

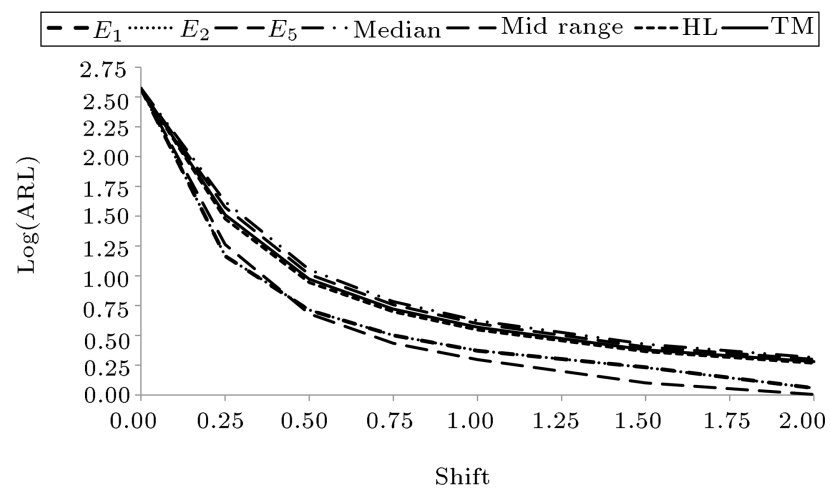

Figure 2. Average Run Length (ARL) curves of the proposed CUSUM charts using $E_{j}(j=1,2,5)$ and the existing CUSUM charts based on median, mid-range, HL, and trimean when $n=5, k=0.5$, and $\rho_{x y}=0.75$ at $A R L_{0}=370$.

the superiority of the proposed CUSUM charts over the existing CUSUM-Mean and CUSUM-FIR charts for $n=5, k=0.50$, and $\rho_{x y}=0.90$ at $A R L_{0}=370$. However, Figure 2 demonstrates the supremacy of the suggested CUSUM control charts over robust CUSUM charts introduced by Nazir et al. [37] for $n=5$, $k=0.50$, and $\rho_{x y}=0.75$ at $A R L_{0}=370$.

\subsection{Illustrative example}

To show the sensitivity of the control charts under investigation, an example is provided that compares the proposed CUSUM control charts based on ratiotype estimators $E_{j}(j=1,2, \cdots, 5)$ with the existing CUSUM mean chart. The target variable $(Y)$ and the auxiliary variable $(X)$ may be respectively defined as follows:

(i) Single-strand break factor (a measure of breaking strength) and weight of textile fibers (hanks per pound) in the fiber production process;

(ii) Tensile strength (psi) and the amount of molybdenum in the production industry of steel wire; (iii) The amount of power generated (MW) and the amount of flue gas in the power generation sector;

(iv) The pharmaceutical product and temperature in the pharmaceutical industry.

Some real-life applications of the use of auxiliary variables in control charts were provided by Ahmad et al. [38].

To demonstrate the detection ability of the proposed charts, 35 samples, characterized by size 10 each, are generated from a bivariate normal distribution with $\rho_{x y}=0.75$. The first 20 samples are generated from the in-control bivariate normal process with $\boldsymbol{\mu}=\left[\begin{array}{ll}4 & 4\end{array}\right]$ and $\boldsymbol{\Sigma}=\left(\begin{array}{cc}1 & 0.75 \\ 0.75 & 1\end{array}\right)$, and the last 15 samples are also generated from a shifted bivariate normal distribution, where a mean shift of magnitude $\delta=0.25$ is introduced in the study variable. Under a fixed $A R L_{0}=370$, the upper-CUSUM charts based on the mean and the ratio estimator $E_{1}$ with parameters $k=$ 0.5 and $H_{1} \cong H=4.77$ are constructed and shown in Figure 3. Moreover, the monitoring statistics $\left(M_{j t}^{+}, j=\right.$ $2,3,4,5)$ based on the estimators $E_{j}(j=2,3,4,5)$ are plotted against the decision interval $H_{j}(j=2,3,4,5)$ to guarantee that $A R L_{0}=370$. The respective control charts are presented in Figures 4 and 5 .

From Figures 3-5, it is apparent that the CUSUM charts based on the mean and the estimators

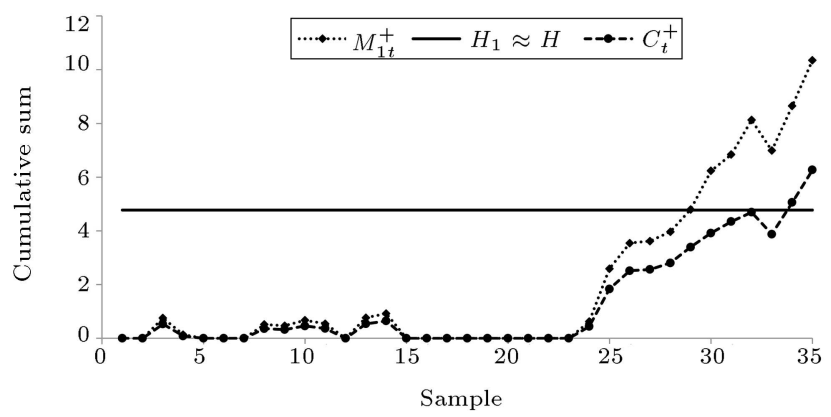

Figure 3. Upper-CUSUM chart based on the mean and the ratio estimator $E_{1}$ for the simulated data using $k=0.5$ and $\rho_{x y}=0.75$ at $n=10$ and $A R L_{0}=370$.

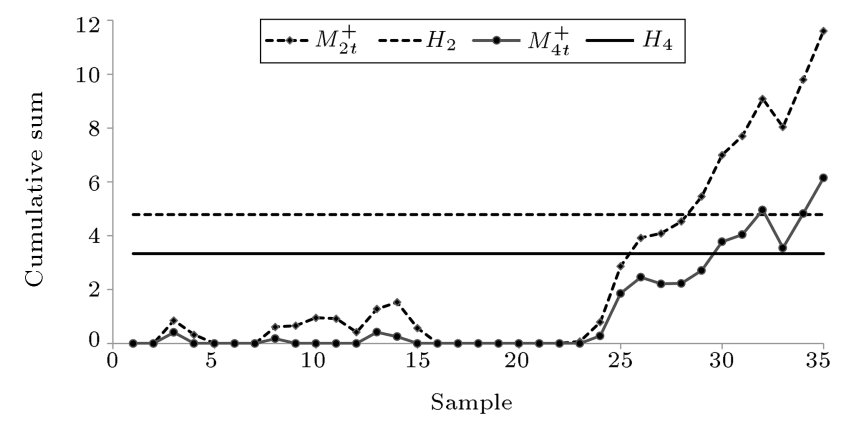

Figure 4. Upper-CUSUM chart based on the ratio type estimators $E_{2}$ and $E_{4}$ for the simulated data using $k=0.5$ and $\rho_{x y}=0.75$ at $n=10$ and $A R L_{0}=370$. 


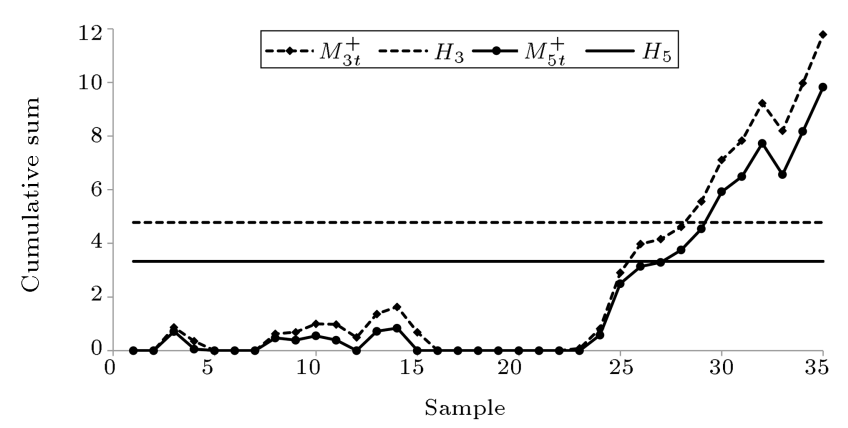

Figure 5. Upper-CUSUM chart based on the ratio type estimator $E_{3}$ and $E_{5}$ for the simulated data using $k=0.5$ and $\rho_{x y}=0.75$ at $n=10$ and $A R L_{0}=370$.

$E_{j}(j=1,2, \cdots, 5)$ trigger the out-of-control signals in Samples 34-35, 29-35, 29-35, 29-35, 30-35, and 28-35, respectively. The results indicate that the proposed control charts detect the shift in the process mean more rapidly than the existing CUSUM mean chart. According to Figures 3-5, the CUSUM chart based on $E_{5}$ requires eight samples to detect a mean shift, which is faster than other charts. This example clearly reveals the superiority of the proposed charts over the existing charts.

\section{Conclusion}

In this study, new CUSUM control charts were proposed for monitoring the location of the normal processes. The scheme incorporated the auxiliary information to estimate the process mean by making advantageous use of the correlation between the auxiliary and the study variables. The suggested charts modify Robert's CUSUM chart by replacing the sample mean with more efficient ratio-type estimators of the mean. In general, the proposed CUSUM charts based on $E_{2}$ and $E_{3}$ had smaller $A R L_{1} \mathrm{~s}$, SDRLs, and EQL under a moderate correlation than other charts. In case of a high correlation, the CUSUM chart based on $E_{1}$ gave smaller $A R L_{1}$ s for small mean shifts $(\delta \leq 0.25)$, whereas the CUSUM chart based on $E_{5}$ produced better results in terms of $A R L_{1} \mathrm{~s}$ for larger mean shifts $(\delta>0.25)$. The superiority of the new CUSUM control charts was confirmed by comparing the outof-control ARLs of the various existing charts with the proposed charting schemes using a range of mean shifts, sample sizes, and different levels of correlation. An illustrative example was also given to assess the performance of the proposed charts, which showed that the proposed charts detected the process shift more quickly. The sensitivity of the proposed charts increased with an increase in the correlation between the study and auxiliary variables. The present work can be further extended to design other control charts under the improved form of estimators based on the auxiliary information.

\section{Acknowledgments}

The authors are deeply grateful to the editor and reviewers for their suggestions to improve the quality of the paper.

\section{Funding}

This research work was partially supported by the National Natural Science Foundation of China (11531001).

\section{References}

1. Montgomery, D.C., Introduction to Statistical Quality Control, Wiley New York, 7th Edn (2012).

2. Page, E.S. "Continuous inspection schemes", Biometrika, 41(1/2), pp. 100-115 (1954).

3. Roberts, S.W. "Control chart tests based on geometric moving averages", Technometrics, 1(3), pp. 239-250 (1959).

4. Haq, A. and Shabbir, J. "Improved family of ratio estimators in simple and stratified random sampling", Communications in Statistics-Theory and Methods, 42(5), pp. 782-799 (2013).

5. Kadilar, C. and Cingi, H. "Ratio estimators in simple random sampling", Applied Mathematics and Computation, 151(3), pp. 893-902 (2004).

6. Kadilar, C. and Cingi, H. "New ratio estimators using correlation coefficient", Interstat, 4, pp. 1-11 (2006).

7. Gupta, S. and Shabbir, J. "On improvement in estimating the population mean in simple random sampling", Journal of Applied Statistics, 35(5), pp. 559566 (2008).

8. Al-Omari, A.I. "Ratio estimation of the population mean using auxiliary information in simple random sampling and median ranked set sampling", Statistics \& Probability Letters, 82(11), pp. 1883-1890 (2012).

9. Grover, L.K. and Kaur, P. "A generalized class of ratio type exponential estimators of population mean under linear transformation of auxiliary variable", Communications in Statistics-Simulation and Computation, 43(7), pp. 1552-1574 (2014).

10. Singh, H.P., Pal, S.K., and Solanki, R.S. "A new class of estimators of finite population mean in sample surveys", Communications in Statistics-Theory and Methods, 46(6), pp. 2630-2637 (2017).

11. Irfan, M., Javed, M., and Lin, Z. "Efficient ratiotype estimators of finite population mean based on correlation coefficient", Scientia Iranica, 25(4), pp. 2361-2372 (2018).

12. Javed, M., Irfan, M., Pang, T., and Lin, Z. "On improved estimation of population mean using known coefficient of skewness of an auxiliary variable", Iranian Journal of Science and Technology, Transactions A: Science, pp. 1-11 (2018).

DOI: $10.1007 / \mathrm{s} 40995-018-0561-5$ 
13. Zhang, G. "Cause-selecting control charts-a new type of quality control charts", The QR Journal, 12(4), pp. 221-225 (1985).

14. Riaz, M. "Monitoring process mean level using auxiliary information", Statistica Neerlandica, 62(4), pp. 458-481 (2008).

15. Riaz, M. "Monitoring process variability using auxiliary information", Computational Statistics, 23(2), pp. 253-276 (2008).

16. Abbas, N., Riaz, M., and Does, R.J. "An EWMA-type control chart for monitoring the process mean using auxiliary information", Communications in StatisticsTheory and Methods, 43(16), pp. 3485-3498 (2014).

17. Ahmad, S., Abbasi, S.A., Riaz, M., and Abbas, N. "On efficient use of auxiliary information for control charting in SPC", Computers \& Industrial Engineering, 67, pp. $173-184$ (2014).

18. Riaz, M. "Control charting and survey sampling techniques in process monitoring", Journal of the Chinese Institute of Engineers, 38(3), pp. 342-354 (2015).

19. Hussain, S., Song, L., Ahmad, S., and Riaz, M. "On auxiliary information based improved EWMA median control charts", Scientia Iranica, 25(2), pp. 954-982 (2018).

20. Lee, H., Aslam, M., Shakeel, Q.-A., Lee, W., and Jun, C.-H. "A control chart using an auxiliary variable and repetitive sampling for monitoring process mean", Journal of Statistical Computation and Simulation, 85(16), pp. 3289-3296 (2015).

21. Riaz, M. "An improved control chart structure for process location parameter", Quality and Reliability Engineering International, 27(8), pp. 1033-1041 (2011).

22. Riaz, M., Mehmood, R., Ahmad, S., and Abbasi, S.A. "On the performance of auxiliary-based control charting under normality and nonnormality with estimation effects", Quality and Reliability Engineering International, 29(8), pp. 1165-1179 (2013).

23. Haq, A. "New EWMA control charts for monitoring process dispersion using auxiliary information", Quality and Reliability Engineering International, 33(8), pp. 2597-2614 (2017).

24. Arshad, W., Abbas, N., Riaz, M., and Hussain, Z. "Simultaneous use of runs rules and auxiliary information with exponentially weighted moving average control charts", Quality and Reliability Engineering International, 33(2), pp. 323-336 (2017).

25. Raza, S.M.M. and Butt, M.M. "New Shewhart and EWMA type control charts using exponential type estimator with two auxiliary variables under two phase sampling", Pakistan Journal of Statistics and Operation Research, 14(2), pp. 367-386 (2018).

26. Hussain, S., Song, L., Ahmad, S., and Riaz, M. "New interquartile range EWMA control charts with applications in continuous stirred tank rector process",
Arabian Journal for Science and Engineering, 44, pp. 2467-2485 (2019). https://doi.org/10.1007/s13369-018-3162-x

27. Saghir, A., Ahmad, L., Aslam, M., and Jun, C.-H. "A EWMA control chart based on an auxiliary variable and repetitive sampling for monitoring process location", Communications in Statistics-Simulation and Computation, 48(7), pp. 2034-2045 (2019). DOI: $10.1080 / 03610918.2018 .1433837$

28. Sanusi, R.A., Riaz, M., and Abbas, N. "Combined Shewhart CUSUM charts using auxiliary variable", Computers \& Industrial Engineering, 105, pp. 329337 (2017).

29. Cochran, W.G., Sampling Techniques, John Wiley \& Sons, 3rd Edn. (2007).

30. Qiu, P., Introduction to Statistical Process Control, CRC Press (2014).

31. TEAM, R.C. "R: A language and environment for statistical computing", $R$ Foundation for Statistical Computing, Vienna, Austria, ISBN 3-900051-07-0 (2017), URL: http://www.R-project.org.

32. Abujiya, M.R., Lee, M.H., and Riaz, M. "Increasing the sensitivity of cumulative sum charts for location", Quality and Reliability Engineering International, 31(6), pp. 1035-1051 (2015).

33. Wu, Z., Yang, M., Jiang, W., and Khoo, M.B.C. "Optimization designs of the combined ShewhartCUSUM control charts", Computational Statistics \& Data Analysis, 53(2), pp. 496-506 (2008).

34. Ou, Y., Wu, Z., and Tsung, F. "A comparison study of effectiveness and robustness of control charts for monitoring process mean", International Journal of Production Economics, 135(1), pp. 479-490 (2012).

35. Nawaz, T., Raza, M.A., and Han, D. "A new approach to design efficient univariate control charts to monitor the process mean", Quality and Reliability Engineering International, 34(8), pp. 1732-1751 (2018). DOI: 10.1002 /qre.2366

36. Lucas, J.M. and Crosier, R.B. "Fast initial response for CUSUM quality-control schemes: give your CUSUM a head start", Technometrics, 24(3), pp. 199-205 (1982).

37. Nazir, H.Z., Riaz, M., Does, R.J.M.M., and Abbas, Nasir. "Robust CUSUM control charting", Quality Engineering, 25(3), pp. 211-224 (2013).

38. Ahmad, S., Riaz, M., Abbasi, S.A., and Lin, Z. "On efficient median control charting", Journal of the Chinese Institute of Engineers, 37(3), pp. 358-375 (2014).

\section{Biographies}

Muhammad Ali Raza obtained his MSc and MPhil degrees in Statistics from the University of the Punjab Lahore, Pakistan. He has been working as an Assistant Professor at the Department of Statistics, Government College University Faisalabad, Pakistan since October 
2011. Currently, he is pursuing his $\mathrm{PhD}$ (Statistics) from the School of Mathematical Sciences, Shanghai Jiao Tong University, Shanghai, the People's Republic of China under the Chinese Government Scholarship Program (2015). His research interest includes statistical process control and applied statistics.

Tahir Nawaz obtained his MSc and MPhil degrees in Statistics from the Islamia University Bahawalpur, Pakistan and Government College University Lahore, Pakistan, respectively. He served as a Statistical Officer in Punjab Bureau of Statistics from May 2007 to November 2009. He served as a Lecturer in Statistics in the Islamia University Bahawalpur, Pakistan from November 2009 to October 2013. He is working as a Lecturer at the Department of Statistics, Government College University Faisalabad, Pakistan since November 2013. Currently, he is pursuing his PhD in Statistics from the School of Mathematical Sciences, Shanghai Jiao Tong University, Shanghai, the People's Republic of China under the Chinese Government Scholarship Program (2016). His research interest includes statistical process control, distribution theory, and survey sampling.

Muhammad Aslam earned his MSc in Statistics (2004) from GC University Lahore with Chief Minister of the Punjab merit scholarship, MPhil in Statistics (2006) from GC University Lahore with the Governor of the Punjab merit scholarship, and $\mathrm{PhD}$ in Statistics (2010) from National College of Business Administration \& Economics Lahore under the kind supervision of Prof. Dr. Munir Ahmad. He worked as a Lecturer of Statistics in Edge College System International College from 2003-2006. He also worked as a Research Assistant at the Department of Statistics, GC University Lahore from 2006 to 2008. Then, he joined the Forman Christian College University as a Lecturer in August 2009. He worked as an Assistant Professor at the same University from June 2010 to April 2012. He worked at the same department as Associate Professor from June 2012 to October 2014. He worked as an Associate Professor of Statistics at the Department of Statistics, Faculty of Science, King Abdulaziz University, Jeddah, Saudi Arabia from October 2014 to March 2017. He taught a summer course as Visiting Faculty of Statistics at Beijing Jiaotong University, China in 2016. Currently, he is working as a Full Professor of Statistics at the Department of Statistics, King Abdul-Aziz University Jeddah, Saudi Arabia. He has published more than 265 research papers in well-reputed national and international journals including IEEE Access, Journal of Applied Statistics, European Journal of Operation
Research, Information Sciences, Journal of Process Control, Journal of the Operational Research Society, Applied Mathematical Modeling, International Journal of Advanced Manufacturer Technology, Communications in Statistics, Journal of Testing and Evaluation and Pakistan Journal of Statistics, etc. His papers have been cited more than 2100 times with h-index 25 and i-10 index 64 (Google Coalitions). His papers have been cited more than 1000 times with h-index 18 (Web of Science Coalitions). He is the author of one book published in Germany. $\mathrm{He}$ is also HEC approved $\mathrm{PhD}$ supervisor since 2011. He supervised $5 \mathrm{PhD}$ theses, more than $25 \mathrm{MPhil}$ theses, and $3 \mathrm{MSc}$ theses. Dr. Aslam is currently supervising $1 \mathrm{PhD}$ thesis and more than 5 MPhil theses in statistics. He is a reviewer of more than 50 well reputed international journals. He has reviewed more than 140 research papers for various well-reputed international journals. He received a meritorious services award in research from National College of Business Administration \& Economics Lahore in 2011. He received the Research Productivity Award for the year 2012 by Pakistan Council for Science and Technology. His name is listed at the 2nd position among statisticians in the Directory of Productivity Scientists of Pakistan in 2013. His name is listed at the 1st position among statisticians in the Directory of Productivity Scientists of Pakistan, 2014. He obtained 371 positions in the list of top 2210 profiles of Scientist of Saudi Institutions, 2016. $\mathrm{He}$ is selected for "Innovative Academic Research \& Dedicated Faculty Award 2017" by SPE, Malaysia. He received King Abdulaziz University Excellence Awards in Scientific Research for the paper entitled "Aslam, M., Azam, M., Khan, N. and Jun, C.-H. (2015). A New Mixed Control Chart to Monitor the Process, International Journal of Production Research, 53 (15), 4684-4693. He received King Abdulaziz University citation award for the paper entitled "Azam, M., Aslam, M. and Jun, C.-H. (2015). He designed a hybrid exponentially weighted moving average control chart using repetitive sampling, International Journal of Advanced Manufacturing Technology, 77:1927-1933 in 2018. He is a member of the editorial board of Electronic Journal of Applied Statistical Analysis, Asian Journal of Applied Science and Technology and Pakistan Journal of Commerce and Social sciences. $\mathrm{He}$ is also a member of Islamic Countries Society of Statistical Sciences. He is appointed as an external examiner for 2016/2017-2018/2019 triennium at The University of Dodoma, Tanzania. His areas of interest include reliability, decision trees, industrial statistics, acceptance sampling, rank set sampling, neutrosophic statistics, and applied statistics. 